University of Nebraska - Lincoln

DigitalCommons@University of Nebraska - Lincoln

3-13-2009

\title{
Plateau Structure in Resonant Laser-Assisted Electron-Atom Scattering
}

A. V. Flegel

Voronezh State University, Russia

M. V. Frolov

Voronezh State University, Russia

N. L. Manakov

Voronezh State University, manakov@phys.vsu.ru

Anthony F. Starace

University of Nebraska-Lincoln, astarace1@unl.edu

Follow this and additional works at: https://digitalcommons.unl.edu/physicsstarace

Part of the Physics Commons

Flegel, A. V.; Frolov, M. V.; Manakov, N. L.; and Starace, Anthony F., "Plateau Structure in Resonant LaserAssisted Electron-Atom Scattering" (2009). Anthony F. Starace Publications. 166.

https://digitalcommons.unl.edu/physicsstarace/166

This Article is brought to you for free and open access by the Research Papers in Physics and Astronomy at DigitalCommons@University of Nebraska - Lincoln. It has been accepted for inclusion in Anthony F. Starace Publications by an authorized administrator of DigitalCommons@University of Nebraska - Lincoln. 


\title{
Plateau Structure in Resonant Laser-Assisted Electron-Atom Scattering
}

\author{
A. V. Flegel, ${ }^{1}$ M. V. Frolov, ${ }^{2}$ N. L. Manakov, ${ }^{2}$ and Anthony F. Starace ${ }^{3}$ \\ ${ }^{1}$ Department of Computer Science, Voronezh State University, Voronezh 394006, Russia \\ ${ }^{2}$ Department of Physics, Voronezh State University, Voronezh 394006, Russia \\ ${ }^{3}$ Department of Physics and Astronomy, The University of Nebraska, Lincoln, Nebraska 68588-0111, USA
}

(Received 15 October 2008; published 11 March 2009)

\begin{abstract}
Orders of magnitude increases are predicted in the cross sections for electron-atom scattering accompanied by absorption or emission of $n$ laser photons for incident electron energies at which the electron, by emitting $\mu$ laser photons, can be captured by the atom to form a negative ion. Enhancements are most significant in the plateau region $(n \gg \mu)$ of the scattered electron spectrum, whose shape is predicted to replicate that of the ion's $(n+\mu)$-photon detachment spectrum.
\end{abstract}

PACS numbers: 34.80.Qb, 03.65.Nk, 32.80.Gc

Nearly constant cross sections (as a function of the number of absorbed photons) have been predicted recently over a large range of scattered electron energies in laserassisted electron-atom scattering (LAES) for both linear [1] and circular [2] laser polarizations. Such "plateau" structures have been investigated for over two decades in other intense laser processes, e.g., above-threshold ionization or detachment (ATI or ATD) and high-order harmonic generation (cf. the review [3]). Interest in such plateaus centers on the possibility of transferring large amounts of energy from a laser field into either electron kinetic energy or high-order harmonics without significant decreases in yields. However, for all processes the absolute values of the $n$-photon rates in the plateau region are orders of magnitude smaller than in the region of small $n$. Hence, mechanisms for increasing rates on the plateau are of great interest. For LAES, two such mechanisms exist: thresholdrelated phenomena at the closing of the stimulated multiphoton emission channel [4] and resonance scattering. These two mechanisms occur under different conditions: the former occur at incident electron energies equal to multiples of the photon energy, while the latter occurs for those electron energies at which the electron is temporarily captured (following stimulated emission of $\mu$ laser photons) in a bound state of the potential $U(r)$ of the target atom (or positive ion) and then detached (or ionized) by absorbing $n+\mu$ photons. Resonant LAES in an intense circularly polarized laser field was considered in Ref. [5], modeling $U(r)$ by a zero-range potential. A series of resonances in $e-\mathrm{H}^{+}$scattering in a circularly polarized laser field were found in Refs. [6,7]. However, the existence of resonant enhancements in high-energy LAES spectra, as well as the influence of resonance effects on the shape of the high-energy plateau in LAES, remain open questions.

In this Letter we predict significant resonant enhancements of plateau features in LAES for the case of electron scattering from neutral atoms supporting negative ions and show that the shape of the high-energy plateau in resonant LAES spectra coincides with that for ATD.
We consider electron-atom scattering in a laser electric field $\mathbf{F}(t)=\mathbf{F} \cos \omega t$ for an incident electron energy, $E=$ $p^{2} / 2 m$, that is small compared to atomic excitation energies and for laser parameters such that laser excitation or ionization of atomic electrons is negligible. Under these assumptions, the process can be described as lasermodified elastic electron scattering from an atomic potential $U(r)$, in which the electron absorbs $(n>0)$ or emits $(n<0)|n|$ laser photons, changing its momentum $p$ to $p_{n}=\sqrt{2 m(E+n \hbar \omega)}$ (where $\left.n_{\min }=-[E /(\hbar \omega)]\right)$. The potential $U(r)$ (of radius $r \approx r_{c}$ ) is assumed to support a weakly bound (negative ion) state $\psi_{\kappa l m}(\mathbf{r})$ with energy $E_{0}=-\hbar^{2} \kappa^{2} / 2 m$ and angular momentum $l=0$ ("s scattering") or $l=1$ (" $p$ scattering"), as in $e-\mathrm{H}$ or $e$-F scattering. Since resonant phenomena disappear in a perturbative treatment of the potential $U(r)$, both the electron-atom and electron-laser interactions must be treated nonperturbatively. For this purpose, we employ the time-dependent effective range (TDER) theory [4], which extends effective range theory (ERT) [8] for lowenergy elastic electron scattering from a short-range potential $U(r)$ to the case of LAES. Thus the main approximation in the TDER theory is the same as in ERT: the interaction of an incident electron with an atomic potential $U(r)$ is taken into account only in the $l$-wave channel, by means of the scattering phase $\delta_{l}(E)$, which is parametrized in terms of the scattering length $a_{l}$ and the effective range $r_{l}$. However, TDER theory provides an essentially exact account of electron-laser interactions by using the Green function, $G\left(\mathbf{r}, t ; \mathbf{r}^{\prime}, t^{\prime}\right)$, of an incoming electron in the field $\mathbf{F}(t)$. As a result, the scattering state, $\Phi_{E}(\mathbf{r}, t)$, of the electron within TDER theory has an analytic form in terms of $G\left(\mathbf{r}, t ; \mathbf{r}^{\prime}, t^{\prime}\right)$ and a periodic function, $f_{\mathbf{p}}(t)$, that enters the boundary condition for $\Phi_{E}(\mathbf{r}, t)$ inside the potential well $U(r)$ (i.e., for $r \leq r_{c}$ ). It contains complete information on the modification of the electron-atom interaction by the laser field [4].

The $n$-photon LAES cross section in TDER theory,

$$
d \sigma_{n} / d \Omega_{\mathbf{p}_{n}}=\kappa^{-2}\left(p_{n} / p\right)\left|\mathcal{A}_{n}^{(l)}\left(\mathbf{p}, \mathbf{p}_{n}\right)\right|^{2}
$$


is different for $l=0$ and 1 [4]. We give here only those results for $s$ scattering needed for later discussions (cf. Ref. [4] for the case of $p$ scattering). For $l=0$,

$$
\mathcal{A}_{n}^{(s)}\left(\mathbf{p}, \mathbf{p}_{n}\right)=\sum_{s=-\infty}^{\infty} f_{s}(\mathbf{p}) c_{s-n}^{*}\left(\mathbf{p}_{n}\right),
$$

where $c_{s}(\mathbf{p})$ are the Fourier coefficients of the periodic (in time) part of the well-known (Volkov) wave function for a free electron in a laser field, $\psi_{\mathbf{p}}(\mathbf{r}=\mathbf{0}, t)$ :

$$
c_{s}(\mathbf{p})=i^{s} \mathcal{J}_{-s}\left(\frac{|e| \mathbf{F} \cdot \mathbf{p}}{m \hbar \omega^{2}}, \frac{u_{p}}{2 \hbar \omega}\right),
$$

where $\mathcal{J}_{n}(\alpha, \beta)=\sum_{k} J_{n+2 k}(\alpha) J_{k}(\beta)$ is the generalized Bessel function and $u_{p}=\left(e^{2} F^{2}\right) /\left(4 m \omega^{2}\right)$ is the ponderomotive energy. The Fourier coefficients $f_{s}(\mathbf{p})$ of the function $f_{\mathbf{p}}(t)$ satisfy a system of linear inhomogeneous equations, which separate into two uncoupled systems, one for the coefficients $f_{s} \equiv f_{2 k+\delta}$ with even subscripts $s(\delta=0)$ and the other for $f_{s}$ with odd $s$ $(\delta=1)$ :

$$
\sum_{k^{\prime}} \mathcal{M}_{k, k^{\prime}}\left(E_{\delta}\right) f_{2 k^{\prime}+\delta}(\mathbf{p})=c_{2 k+\delta}(\mathbf{p})
$$

where $E_{p}=E+p \hbar \omega$ and $\mathcal{M}_{k, k^{\prime}}(E)=-M_{k, k^{\prime}}(E)-\{1+$ $\left.i \sqrt{\left(E_{2 k}+u_{p}\right) /\left|E_{0}\right|}-\frac{1}{2} \kappa r_{0}\left[\left(E_{2 k}+u_{p}\right) /\left|E_{0}\right|+1\right]\right\} \delta_{k, k^{\prime}}$. The matrix elements $M_{k, k^{\prime}}(E)$ satisfy the symmetry relation, $M_{k+l, k^{\prime}+l}(E)=M_{k, k^{\prime}}\left(E_{2 l}\right)$, and can be expressed in terms of integrals of Bessel functions (cf. Ref. [1]).

To extract the resonant part of the amplitude (2) explicitly, we note that the LAES amplitude has poles in the plane of complex quasienergy $\epsilon\left(\epsilon=E+u_{p}\right)$ at $\epsilon=$ $\tilde{E}_{0}+p \hbar \omega \quad(p=0, \pm 1, \ldots)$, where $\operatorname{Re} \tilde{E}_{0}$ and $\Gamma=$ $-2 \operatorname{Im} \tilde{E}_{0} / \hbar$ determine the position and total decay rate of the quasistationary quasienergy state (QQES), $\Phi_{\tilde{E}_{0}}(\mathbf{r}, t)$, to which the bound state $\psi_{\kappa l m}(\mathbf{r})$ evolves in a laser field [9]. The complex quasienergy $\tilde{E}_{0}$ is the eigenvalue of the homogeneous equation that follows from Eq. (4) upon substituting $c_{2 k+\delta}=0, E_{\delta} \rightarrow \tilde{E}_{0}-u_{p}$, and $f_{2 k^{\prime}+\delta}(\mathbf{p}) \rightarrow$ $\tilde{f}_{2 k^{\prime}}$ [10]. For the case of a $\mu$-photon resonance, a detailed analysis of Eq. (4) for $E \approx \operatorname{Re} \mathcal{E}_{\mu}$, where $\mathcal{E}_{\mu} \equiv \tilde{E}_{0}-u_{p}+$ $\mu \hbar \omega$, finds a solution for the resonant parts of the coefficients $f_{2 k+\delta}(\mathbf{p})$ in terms of the Fourier coefficients, $\tilde{f}_{2 k}$, of the QQES problem,

$$
f_{2 k+\delta}^{(\mathrm{res})}(\mathbf{p})=\frac{\tilde{f}_{2 k+\delta+\mu}}{N\left(\tilde{E}_{0}\right)\left(E-\mathcal{E}_{\mu}\right)} \sum_{k^{\prime}=-\infty}^{\infty} \tilde{f}_{2 k^{\prime}} c_{2 k^{\prime}-\mu}(\mathbf{p}),
$$

where $\delta$ and $\mu$ have the same parity [i.e., only coefficients $f_{s}$ with even (odd) $s$ contribute to the resonant amplitude for the case of even (odd) $\mu$ ]. The factor $N\left(\tilde{E}_{0}\right)$ is

$$
N\left(\tilde{E}_{0}\right)=\left.\sum_{k, k^{\prime}} \tilde{f}_{2 k} \tilde{f}_{2 k^{\prime}}\left(\partial \mathcal{M}_{k, k^{\prime}}(E) / \partial E\right)\right|_{E=\tilde{E}_{0}-u_{p}} .
$$

Separating Eq. (2) into potential and resonant parts, i.e., $\mathcal{A}_{n}^{(s)}\left(\mathbf{p}, \mathbf{p}_{n}\right)=\mathcal{A}_{n}^{(\mathrm{pot})}+\mathcal{A}_{n}^{(\mathrm{res})}$, and using Eq. (5) in the latter, its dependence on $\mathbf{p}$ and $\mathbf{p}_{n}$ factorizes:

$$
\begin{gathered}
\mathcal{A}_{n}^{(\mathrm{res})}\left(\mathbf{p}, \mathbf{p}_{n}\right)=\frac{\mathcal{A}_{\mu}^{(\mathrm{at})}(\mathbf{p}) \mathcal{A}_{n+\mu}^{(\mathrm{det})}\left(\mathbf{p}_{n}\right)}{N\left(\tilde{E}_{0}\right)\left(E-\mathcal{E}_{\mu}\right)}, \\
\mathcal{A}_{\mu}^{(\mathrm{at})}(\mathbf{p})=\sum_{k} \tilde{f}_{2 k} c_{2 k-\mu}(\mathbf{p}), \\
\mathcal{A}_{n+\mu}^{(\mathrm{det})}\left(\mathbf{p}_{n}\right)=\sum_{k} \tilde{f}_{2 k} c_{2 k-(n+\mu)}^{*}\left(\mathbf{p}_{n}\right) .
\end{gathered}
$$

The amplitude $\mathcal{A}_{n}^{\text {(res) }}\left(\mathbf{p}, \mathbf{p}_{n}\right)$ clearly describes the resonant channel of $n$-photon LAES as a two-step process: (1) The amplitude $\mathcal{A}_{\mu}^{\text {(at) }}(\mathbf{p})$ does not depend on $n$ and describes the emission of $\mu$ photons by the incident electron followed by its attachment to the atom to form a negative ion whose outer electron is in the QQES, $\Phi_{\tilde{E}_{0}}(\mathbf{r}, t)$. (2) $\mathcal{A}_{n+\mu}^{(\mathrm{det})}\left(\mathbf{p}_{n}\right)$ is the ATD amplitude in TDER theory [10], i.e., it describes the $(n+\mu)$-photon detachment of the electron from the state $\Phi_{\tilde{E}_{0}}(\mathbf{r}, t)$. It is important to emphasize, however, that in multichannel collisions with exchange of an arbitrary number $n$ of laser photons, such as LAES, generally it is difficult to predict in which interval(s) of $n$ the $\mu$-photon resonant amplitude (7) will be dominant compared to the nonresonant (potential) amplitude $\mathcal{A}_{n}^{(\text {pot) }}\left(\mathbf{p}, \mathbf{p}_{n}\right)$. In what follows, we demonstrate the significance of resonant effects in the plateau region of LAES spectra based on both numerical results and analytic estimates of the LAES amplitude.

In Fig. 1 we present numerical results [obtained using the exact amplitude (2)] for $e$ - $\mathrm{H}$ scattering in a $\mathrm{CO}_{2}$-laser field. Comparing LAES spectra in Figs. 1(a) and 1(b) for resonant $\left(E=\operatorname{Re} \mathcal{E}_{\mu}\right)$ and nonresonant energies $(E=$ $\left.\operatorname{Re} \mathcal{E}_{\mu} \pm \Delta\right)$, one sees that the low-energy part of each spectrum is not sensitive to resonant effects, while giant (up to 2 orders of magnitude) resonance enhancements occur on the high-energy plateau and beyond, as well as in LAES with photon emission [cf. Fig. 1(c)]. For scattering at fixed angle $\theta$ between $\mathbf{p}_{n}$ and $\mathbf{F}$, the magnitude of enhancements is sensitive to $n$, i.e., the shape of the plateau exhibits broad oscillations [cf. Fig. 1(a) for $\theta=0$ ]. At the minima of these oscillations, significant destructive interference of resonant and potential amplitudes leads to deep minima in the cross sections. The positions of these minima depend on $\theta$, so that the angle-integrated spectrum is smooth [cf. Fig. 1(b)]. For this case, the effective enhancement factor increases from 10 up to $10^{2}$ as $n$ increases from 50 to 120 . Finally, the results on resonance are sensitive to the spatial symmetry of $\psi_{\kappa l m}(\mathbf{r})$ : for $e$-F scattering, resonant behavior occurs also in the low-energy LAES spectrum [for $n \approx(7-17)$ ], where the nonresonant spectrum has a deep minimum.

The energy dependence of $d \sigma_{n} / d \Omega_{\mathbf{p}_{n}}$ for fixed $n$ near the $\mu$-photon resonances and the thresholds of $k$-photon emission is shown in Fig. 2 for $n$ values at both the 

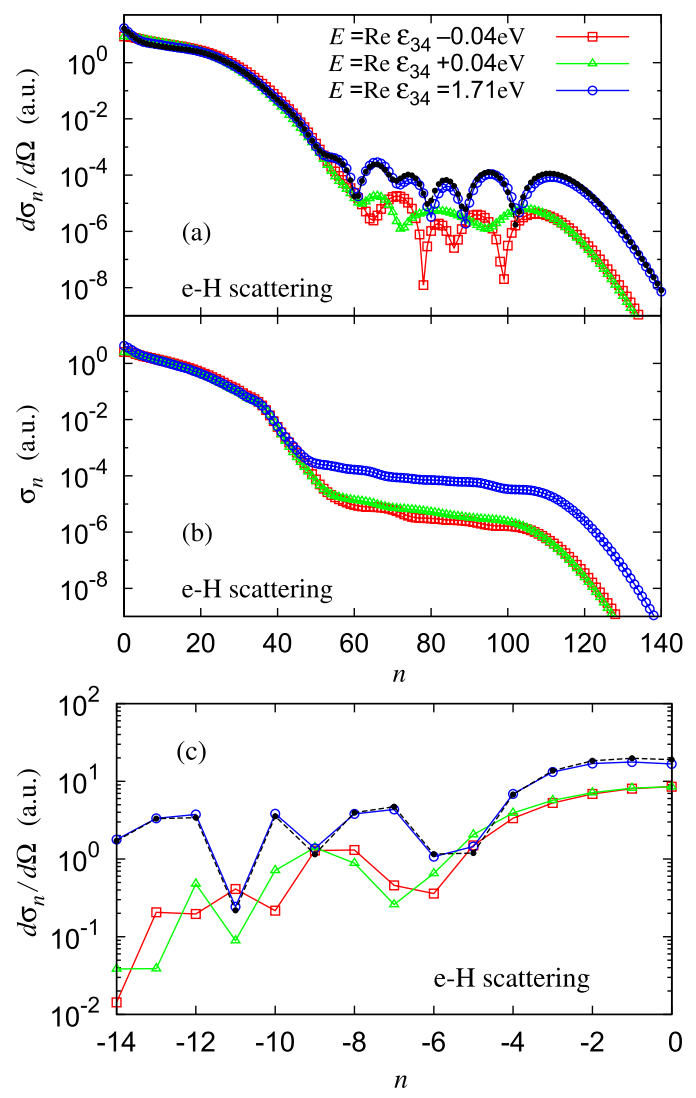

FIG. 1 (color online). LAES spectra for $e$-H scattering in a $\mathrm{CO}_{2}$ laser field $\left(\lambda=10.6 \mu \mathrm{m}, I=1.44 \times 10^{11} \mathrm{~W} / \mathrm{cm}^{2}\right)$ for three electron energies, given in panel (a). (a) Forward scattering: $\mathbf{p}\left\|\mathbf{p}_{n}\right\| \mathbf{F}$; (b) spectrum integrated over the "forwardscattering" hemisphere, $0 \leq \theta \leq 90^{\circ}$; (c) spectrum of $n$-photon stimulated emission $\left(n_{\min }=-14\right)$. Dotted lines with small filled circles: results using approximations (12) [in (a)] and (10) [in (c)].

beginning of the plateau [cf. Fig. 2(a)] and its high-energy end [cf. Fig. 2(b)]. The widths of the curves near resonance are proportional to the decay rate, $\Gamma=-2 \operatorname{Im} \tilde{E}_{0} / \hbar$, of the QQES $\Phi_{\tilde{E}_{0}}(\mathbf{r}, t)$ and thus depend on the laser parameters. For $\mathrm{F}^{-}$and the laser parameters in Fig. 2, $\tilde{E}_{0}=-(3.49+$ $\left.i 8.4 \times 10^{-3}\right) \mathrm{eV}$ [while for $\mathrm{H}^{-}$(cf. Fig. 1), $\tilde{E}_{0}=$ $\left.-\left(0.76+i 5.46 \times 10^{-4}\right) \mathrm{eV}\right]$. One sees that the threshold enhancements of $d \sigma_{n} / d \Omega_{\mathbf{p}_{n}}$ are much lower than the resonant ones. Moreover, the former disappear in the high-energy part of the plateau [4] [cf. Fig. 2(b)].

Comparison of resonant LAES spectra from $\mathrm{H}$ and $\mathrm{F}$ atoms with ATD spectra of the negative ions $\mathrm{H}^{-}$and $\mathrm{F}^{-}$is given in Fig. 3. (The ATD rates, $\Gamma_{n+\mu}$, were calculated using TDER theory [10].) For both $s$ and $p$ scattering the LAES plateau cutoff positions occur at $10 u_{p}$, just as for ATD. Most remarkably, the oscillatory structure of the plateau for LAES coincides with that of ATD.

The physics of resonant LAES and of the exact numerical TDER results in Figs. 1-3 become clearer if the electron-atom interaction is treated more approximately. By neglecting nondiagonal matrix elements in Eq. (4), one

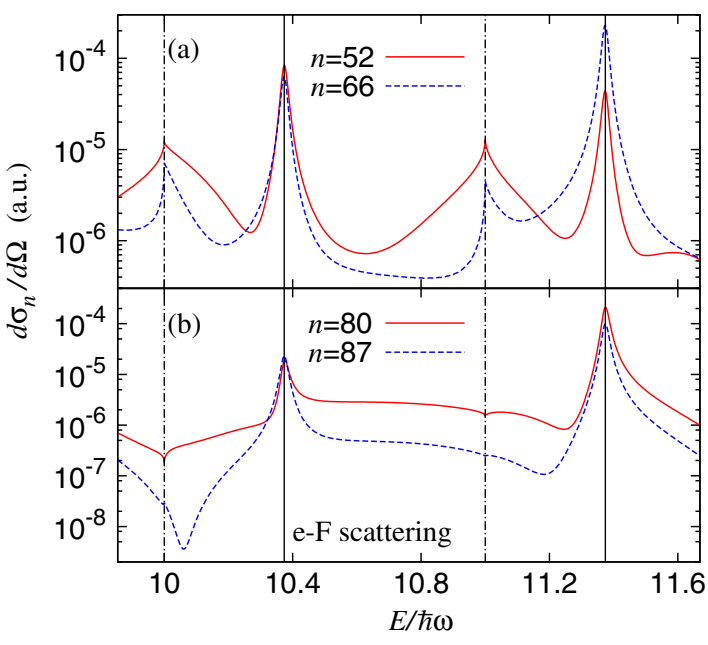

FIG. 2 (color online). Energy dependence of LAES cross sections near the resonant $\left(E=\operatorname{Re} \mathcal{E}_{25}\right.$ and $\left.\operatorname{Re} \mathcal{E}_{26}\right)$ and threshold $(E=10 \hbar \omega$ and $11 \hbar \omega)$ energies for forward $e-\mathrm{F}$ scattering in a laser field for $\lambda=1.8 \mu \mathrm{m}$ and $I=2.19 \times 10^{13} \mathrm{~W} / \mathrm{cm}^{2}$. The vertical solid (dot-dashed) lines mark the resonance (threshold) energies.

accounts for this interaction to lowest order:

$$
f_{s}(\mathbf{p}) \approx f_{s}^{(0)}(\mathbf{p})=c_{s}(\mathbf{p}) / \mathcal{M}_{0,0}\left(E_{s}\right) .
$$

The equation $\mathcal{M}_{0,0}\left(\tilde{E}_{0}-u_{p}\right)=0$ gives the approximate value for $\tilde{E}_{0}, \tilde{E}_{0} \approx \tilde{E}_{0}^{(0)}$. Only the coefficient $f_{-\mu}^{(0)}(\mathbf{p})$ has a pole at the complex energy $E=\tilde{E}_{0}^{(0)}-u_{p}+\mu \omega \equiv \mathcal{E}_{\mu}^{(0)}$ and thus determines $\mathcal{A}_{n}^{(\text {res })}\left(\mathbf{p}, \mathbf{p}_{n}\right)$ near the $\mu$-photon resonance, where

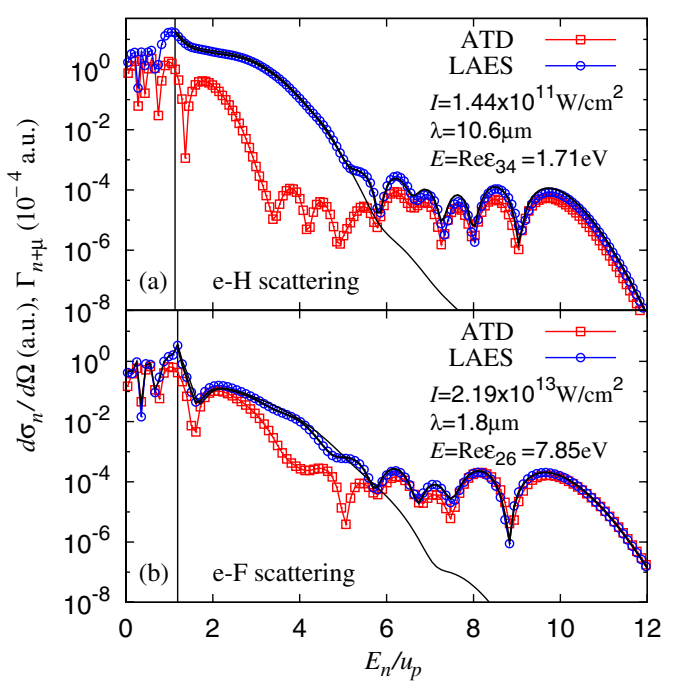

FIG. 3 (color online). Comparison of resonant LAES spectra (a) for $e-\mathrm{H}$ and (b) $e-\mathrm{F}$ forward scattering with the ATD spectra of $\mathrm{H}^{-}$and $\mathrm{F}^{-}$(with detached electron momentum $\mathbf{p}_{n}$ parallel to F). The laser parameters and electron energies are given in panels (a) and (b). The vertical lines correspond to the elastic scattering channel $(n=0)$. The curves represented by the thin and thick lines give the LAES spectrum (for $n \geq 0$ ) calculated using the approximations (10) and (12), respectively. 


$$
\mathcal{M}_{0,0}\left(E_{-\mu}\right) \approx\left(E-\mathcal{E}_{\mu}^{(0)}\right) \mathcal{M}_{0,0}^{\prime},
$$

where $\left.\mathcal{M}_{0,0}^{\prime} \equiv\left(\partial \mathcal{M}_{0,0}(E) / \partial E\right)\right|_{E=\tilde{E}_{0}^{(0)}-u_{p}}$ [cf. Eq. (6)]. Substituting $f_{s}(\mathbf{p}) \rightarrow f_{s}^{(0)}(\mathbf{p})$ in Eq. (2), the amplitude $\mathcal{A}_{n}^{(s)}\left(\mathbf{p}, \mathbf{p}_{n}\right)$ in the approximation (10) [as well as a similar approximation for the $p$-scattering amplitude] reproduces well the exact LAES spectra in Figs. 1-3 below the onset of the plateau (cf. Fig. 3), as well as for negative values of $n$ [cf. Fig. 1(c)]. The dominant contribution of the potential amplitude as compared to the resonant one in the energy region up to the plateau onset explains the somewhat surprising fact that "resonant" and "nonresonant" curves in Figs. 1(a) and 1(b) coincide in this region.

Approximation (10) fails to describe plateaus in LAES spectra owing to its neglect of the coupling between LAES channels with different $n$ [cf. Eq. (4)]. To trace the origin of resonant features in the plateau region, we take into account the nondiagonal matrix elements, $\mathcal{M}_{k, k^{\prime}}\left(=-M_{k, k^{\prime}}\right)$, in Eq. (4) perturbatively. The corresponding correction, $f_{s}^{(1)}$, to $f_{s}^{(0)}$ [i.e., $f_{s} \approx f_{s}^{(0)}+f_{s}^{(1)}$ ] is

$$
f_{s}^{(1)}(\mathbf{p})=\frac{1}{\mathcal{M}_{0,0}\left(E_{s}\right)} \sum_{k \neq 0} M_{0, k}\left(E_{s}\right) f_{2 k+s}^{(0)}(\mathbf{p}),
$$

which corresponds to a more precise account of the potential $U(r)$, beyond the approximation (10). At $E=\mathcal{E}_{\mu}^{(0)}$, $f_{s}^{(1)}(\mathbf{p})$ has poles for both $s=-\mu$ [as does $\left.f_{s}^{(0)}(\mathbf{p})\right]$ and $s=$ $-(\mu+2 k)$. Taking into account the "rescattering correction" (12) to $f_{s}^{(0)}$, the resonant part of the amplitude (2) [cf. Eq. (7)] in the "rescattering approximation" [i.e., $f_{s}(\mathbf{p}) \approx$ $\left.f_{s}^{(0)}(\mathbf{p})+f_{s}^{(1)}(\mathbf{p})\right]$ can be presented as

$$
\begin{aligned}
\mathcal{A}_{n}^{(\mathrm{res})}\left(\mathbf{p}, \mathbf{p}_{n}\right) \approx & \frac{c_{-\mu}(\mathbf{p})}{\left(E-\mathcal{E}_{\mu}^{(0)}\right) \mathcal{M}_{0,0}^{\prime}}\left[c_{-n-\mu}^{*}\left(\mathbf{p}_{n}\right)\right. \\
& \left.+\sum_{k \neq 0} c_{2 k-n-\mu}^{*}\left(\mathbf{p}_{n}\right) \frac{M_{0, k}\left(E_{-\mu}\right)}{\mathcal{M}_{k, k}\left(E_{-\mu}\right)}\right] .
\end{aligned}
$$

Neglecting the sum over $k$ in Eq. (13) yields $\mathcal{A}_{n}^{(\text {res })}\left(\mathbf{p}, \mathbf{p}_{n}\right)$ in the approximation (10).

The "rescattering approximation" for the LAES amplitude is very accurate for describing the resonance plateau features in Figs. 1 and 3, including the destructive interference between $\mathcal{A}_{n}^{\text {(pot) }}$ and $\mathcal{A}_{n}^{\text {(res) }}$ in Fig. 1(a). Equation (13) shows that resonance enhancements of the cross sections $d \sigma_{n} / d \Omega_{\mathbf{p}_{n}}$ (if they exist for a given $n$ ) are governed by the resonant behavior of the $n$-independent, lowest-order Fourier coefficient $f_{-\mu}^{(0)}$ in Eq. (10); the highenergy plateau, however, is described by the nondiagonal matrix elements $M_{0, k}\left(E_{-\mu}\right)$, i.e., it requires a more accurate treatment of electron-atom interaction (similar to that needed to describe plateaus in ATD [10]). Analytic estimates of the matrix elements $M_{0, k}\left(E_{-\mu}\right)$ (similar to those for nonresonant LAES in Ref. [11]) show that the plateau cutoff position for resonant LAES is given by the $10 u_{p}$ law and does not depend on the incoming electron energy $E$.
This $10 u_{p}$ law has the same physical meaning as for ATD [3], i.e., $10 u_{p}$ is the maximum energy acquired by the detached electron after laser-induced rescattering from the parent atom. (Note that the plateau cutoff position for nonresonant LAES depends on $E$ and attains its maximum value, $10 u_{p}$, in the limit $E \rightarrow 0$ [1].)

In conclusion, we have analyzed key features of resonant phenomena in $n$-photon LAES cross sections that occur for incident electron energies corresponding to $\mu$-photon emission and attachment of the incoming electron to the atom. Although resonance effects do not affect significantly the LAES cross sections with absorption of $n \lesssim \mu$ photons, we do find significant resonant enhancements of LAES spectra in the plateau region, for $n \gg \mu$. The remarkable similarity between plateau features in resonant LAES spectra and ATD implies that information about ATD may be obtained from results for resonant LAES and vice versa. These results are useful for better understanding of resonant phenomena in intense laser-atom interactions, as well as for planning of experiments on laser-modified electron-atom scattering. Finally, we note that although resonant enhancements in LAES spectra occur in a narrow interval of electron energies, $\Delta E \lesssim$ (0.04-0.1) eV, electron spectrometers with such highenergy resolution for electron energies of about several $\mathrm{eV}$ have been used in recent experiments [12].

We gratefully acknowledge useful discussions with Timothy J. Gay on experimental matters. This work was supported by RFBR Grants No. 07-02-00574 and No. 0902-00541, by DOE Grant No. DE-FG02-96ER14646, and by Grant No. MK-5585.2008.2 of the President of the Russian Federation (A. V. F.).

[1] N. L. Manakov, A. F. Starace, A. V. Flegel, and M. V. Frolov, Pis'ma Zh. Eksp. Teor. Fiz. 76, 316 (2002) [JETP Lett. 76, 258 (2002)].

[2] A. V. Flegel, M. V. Frolov, N. L. Manakov, and A. F. Starace, Phys. Lett. A 334, 197 (2005).

[3] W. Becker et al., Adv. At. Mol. Opt. Phys. 48, 35 (2002).

[4] N.L. Manakov, A. F. Starace, A. V. Flegel, and M. V. Frolov, Pis'ma Zh. Eksp. Teor. Fiz. 87, 99 (2008) [JETP Lett. 87, 92 (2008)].

[5] I. J. Berson, J. Phys. B 8, 3078 (1975).

[6] L. Dimou and F. H. M. Faisal, Phys. Rev. Lett. 59, 872 (1987).

[7] L. P. Rapoport and A. S. Kornev, Zh. Eksp. Teor. Fiz. 116, 1241 (1999) [Sov. Phys. JETP 89, 664 (1999)].

[8] L.D. Landau and E.M. Lifshitz, Quantum Mechanics (Pergamon, Oxford, 1980), 4th ed.

[9] N. L. Manakov, V.D. Ovsiannikov, and L.P. Rapoport, Phys. Rep. 141, 319 (1986).

[10] M. V. Frolov, N. L. Manakov, E. A. Pronin, and A. F. Starace, Phys. Rev. Lett. 91, 053003 (2003).

[11] A. V. Flegel, M. V. Frolov, N. L. Manakov, and A. F. Starace, J. Phys. B 37, L1 (2004).

[12] M. Allan, Phys. Rev. Lett. 87, 033201 (2001); 93, 063201 (2004). 